\title{
3G Networks in Emergency Telemedicine - An In-Depth Evaluation \& Analysis
}

\author{
Konstantinos Perakis ${ }^{1}$, Konstantinos Banitsas ${ }^{2}$, Georgios Konnis ${ }^{1}$, Dimitris Koutsouris ${ }^{1}$ \\ ${ }^{1}$ Biomedical Engineering Laboratory, National Technical University of Athens, Greece \\ ${ }^{2} \mathrm{E} \& \mathrm{CE}$, School of Engineering and Design, Brunel University, West London, England
}

\begin{abstract}
The evolution of telecommunications technologies in connection with the robustness and the fidelity these new systems provide, have opened up many new horizons as regards the provision of healthcare and the quality of service from the side of the experts to that of the patients. The purpose of this paper is to evaluate the third generation telecommunications systems that are only recently being deployed in Europe, as well as argue on why a transition from $2 \mathrm{G}$ and $2.5 \mathrm{G}$ to $3 \mathrm{G}$ telecommunications systems could prove to be crucial, especially in relation to emergency telemedicine. The experimental results of the use of these systems are analyzed, the implementation of a tele-consultation unit is presented and their exploitation capabilities are explored.
\end{abstract}

Keywords: 3G, GSM, GPRS, Telemedicine, Teleconsultation, Wireless.

\section{INTRODUCTION}

The term telematics, in its wider sense, refers to the application of synchronous telecommunication and information technologies, mainly towards the bidirectional communication by means of audio-visual transmission, targeting the provision of medical care to remote patients, telemetry and the distribution of medical knowledge among doctors. Studies conclude that a constantly increasing number of patients are monitored at home, aiming at the reduction of cost as well as the upgrade of the provided healthcare services [1]. Congestive heart failure, diabetes, asthma as well as high risk pregnancy are only a few of the patient conditions currently included in home telemedicine projects or programs.

According to the Annual Report of the National Centre of Immediate Assistance of Greece, for the year 1998, in 9 out of the 12 evolved precincts, a total of 461,341 ambulance calls took place [2]. In the precinct of Attica (including the island of Evia), a total of 267,076 calls took place, i.e. $57.89 \%$ of the total. Emergency incidents summed up to a total of 371,281 calls, while chronic incidents summed up to a total of 90,060 calls, that translates to 4.12 emergency calls for 1 chronic patient.

All of the above advocate in favor of the need for implementation of innovative telematics products/services via the utilization of communication technologies, new computational systems, and the coding of medical data and biosignals for the development of telemedical devices. One such telemedical device which supported wireless telediagnosis and teleconsultation over GSM networks was a project developed by the National Technical University of Athens in 1998: Emergency 112. The device enabled physicians to provide pre-hospital care more effectively, as critical biosignals (ECG, BP, HR, SpO2, Temperature) and still images of the patients would be sent from the portable device to a base station situated either in a hospital or a medical centre [3]. The deployed GSM networks limit the functionality of the device, providing a bandwidth of up to 9.6 Kbps. Nevertheless, the recently deployed $3 \mathrm{G}$ networks maximize the capabilities of this device and provide the means for more accurate and reliable telediagnosis.

In the following paragraphs the authors attempt to provide the readers with an overview of the wireless networks deployed as far, present them with the results of the trials conducted and emphasize on why the telecommunications evolutions should be indissolubly related to telemedicine.

\section{TECHNICAL OVERVIEW}

GSM: GSM services include telephony, asynchronous and synchronous data services (2.4/4.8/9.6 Kbps), valueadded features (SMS, fax) and more. Speech is digitally encoded and transmitted as a digital stream. GSM users can send and receive data, at rates up to 9,600 bps [4]. Commercial service was started in mid-1991, and by October 1997 GSM subscribers had grown to more than 55 million [5]. The method chosen by GSM to divide up the bandwidth among as many users as possible is a combination of Time- and Frequency-Division Multiple Access (TDMA/FDMA). The FDMA part involves the division by frequency of the (maximum) $25 \mathrm{MHz}$ bandwidth into 124 carrier frequencies spaced $200 \mathrm{kHz}$ apart. One or more carrier frequencies are assigned to each base station. Each of these carrier frequencies is then divided in time, using a TDMA scheme. The fundamental unit of time in this TDMA scheme is called a burst period and lasts 15/26 ms (or approx. $0.577 \mathrm{~ms}$ ). Eight burst periods are grouped into a TDMA frame $(120 / 26 \mathrm{~ms}$, or approx. $4.615 \mathrm{~ms}$ ).

GPRS: GPRS (Short for General Packet Radio Service) was recently introduced as the packet-mode extension to GSM and constitutes the lead-in to $3 \mathrm{G}$ telecommunications technologies [5]. The first GPRS trials were conducted in 1999. The first GPRS networks rolled out in the year 2000. GPRS is a new non-voice, valueadded, high-speed, packet-switching technology for GSM networks. It facilitates sending and receiving small bursts of data as well as large volumes of data over a mobile telephone network. Its main innovations are that it is packet 
based, it increases data transmission speeds, and the user no longer needs to dial up to a separate ISP [6]. GPRS users are considered to be always connected. Packet switching means that GPRS radio resources are used only when users are actually sending or receiving data.

Theoretically, a GPRS connection can provide a data transmission speed of up to $171.2 \mathrm{Kbps}$ (approximately three times as fast as the data transmission speeds of fixed telecommunications networks and ten times as fast as the current GSM network services) if all eight slots are used. However, it is unlikely that network operators will allow a single user use up all the time slots. Nevertheless, bit rates up to $56 \mathrm{Kbps}$ are achievable. The fewer the timeslots provided per user, the lower the data transmission speed.

UMTS: UMTS stands for Universal Mobile Telecommunication System and constitutes Europe's implementation of the 3G Telecommunications Systems. These new systems are a significant innovation over $2 \mathrm{G}$ and $2.5 \mathrm{G}$ systems because of their high operating flexibility, their ability to provide a wide range of applications and generally extend the services now provided to fixed networks users to mobile customers. When conceived, UMTS aspired to provide bit rates up to 2 Mbps to low mobility users and up to $144 \mathrm{Kbps}$ to high mobility users, while being compatible with the 2G GSM system [7].

In order to maximize the number of users served per cell UMTS utilizes the CDMA technique (Code Division Multiple Access) which belongs to the family of spread spectrum techniques [8]. Systems with CDMA access techniques enable users to transmit at the same frequency simultaneously, thus giving the ability to superimpose several signals on the same radio channel.

In CDMA networks, all terminals use the same radio frequency. CDMA systems can provide fully asynchronous hand-over functions as well as the means for active connection maintenance between the mobile terminal and the network, over several radio links, which can also be activated through different radio base stations. In the channel changing stage, typical of the hand-over process, connection continuity is guaranteed through the multiple paths set up between the mobile terminal and the "controlling point" in the network, whereas in GSM for example, channel changing takes place by means of a "hop" between the radio channels of the two cells involved in the process, and hence through an "interruption" whose duration must necessarily be limited. In other words, in order to improve communication quality, the mobile terminal does not limit itself on remaining connected to a single Base Station but involves all Base Stations from which it receives a sufficiently good reference signal in the communication. The process of activating and releasing parallel links is carried out as a function of the quality of the signal received by the mobile terminal as it moves in the network.

\section{METHODOLOGY}

For the evaluation of the $3 \mathrm{G}$ telecommunication network recently deployed by Vodafone Greece, for telemedicine (telediagnosis and telecare) needs, the following tests were conducted and their results are displayed in Tables II-IV. These results are also analyzed both in the results and in the discussion section, where several other scenarios are also taken into consideration. To conduct these tests, the authors used a high-end laptop, a web-cam, a 3G/GPRS PCMCIA card provided by Vodafone GR and a Welch-Allyn medical monitor. The collection of the biosignals was achieved via the use of the Emergency 112 software.

The first series of measurements conducted involved the transmission of critical biosignals, namely the waveforms and the arithmetic data of ECG, Invasive and Non-Invasive Blood Pressure, $\mathrm{SpO} 2$ and Temperature. For the collection of these biosignals the authors used the Welch-Allyn Propaq CS, Model 244 Medical Monitor, with an embedded In-Service Demo [9] as well as an ECG simulator and the appropriate sensors for the collection of the rest of the biosignals. Along with the measurements on $\mathrm{SpO}_{2}$ and $\mathrm{BP}$, the authors transmitted over the air 5 hours of simulated ECG real time and the payload produced did not exceed a few KB [10]. The delay was less than $1 \mathrm{sec}$ during the measurements, thus, the presentation of the results was not reckoned purposeful. What is purposeful is an introduction to the terms bandwidth and throughput, numerically presented as the time required for the transmission of low resolution images in a GSM network. The results of those measurements are presented in Table I [10].

TABLE I

TRANSMISSION OF IMAGES IN GSM NETWORKS

\begin{tabular}{ccc}
\hline File Size (KB) & $\begin{array}{c}\text { Average } \\
\text { Transmission } \\
\text { Time (100 Files) }\end{array}$ & $\begin{array}{c}\text { Throughput } \\
\text { (bps) }\end{array}$ \\
\hline 6 & 18 & 2666.7 \\
7 & 20.5 & 2731.7 \\
8 & 24 & 2666.7 \\
9 & 26 & 2769.7 \\
\hline
\end{tabular}

The second series of measurements conducted involved 20 static images, shot from various distances, from $20 \mathrm{~cm}$ up to $200 \mathrm{~cm}$ away from the patient. These images were taken using a web camera and were being compressed in a JPEG format and segmentally transmitted from the client to the server. The results of these measurements are gathered in Table II. As can be seen, the bigger the size of the image transmitted (the lower the compression and thus the real-time image analysis), the higher the throughput. This fact indicates the ability to transmit uncompressed images introducing a minor time delay. These 20 images taken were compressed in various rates, from $50 \%$ down to $0 \%$ (uncompressed image) using a $5 \%$ compression rate decrease step, adding up to a total of 220 images. 
The third series of tests concerned the transmission of various medical images of different sizes, dimensions, bit depths and color-modes, all stated in Table III. These images were uploaded on a server using the HTTP and the TCP/IP protocols. For reasons of accuracy, the same images were uploaded 10 times each and the results displayed are the average ones. Finally, the fourth series of tests regarded the transmission of the same medical images using the File Transfer Protocol with an FTP server and client. This series of tests was conducted in order to explore the full capabilities of the network in terms of throughput.

\section{RESULTS}

The second series of measurements regarded the transmission of 220 static images of the patient in groups of 20 images each, of different compression rate and thus different payload. The utilization of the network in terms of the average time required for the transmission and the throughput are presented in Table II.

TABLE II

TRANSMISSION OF STATIC IMAGES USING TCP/IP OVER 3G NETWORKS

\begin{tabular}{cccccc}
\hline $\begin{array}{c}\text { C.R } \\
(\%)\end{array}$ & $\begin{array}{c}\text { A.B.T } \\
(\text { Bytes })\end{array}$ & $\begin{array}{c}\text { ATR } \\
(\mathrm{sec})\end{array}$ & $\begin{array}{c}\text { ANU } \\
(\%)\end{array}$ & $\begin{array}{c}\text { A.P.S } \\
(\text { Pac. })\end{array}$ & $\begin{array}{c}\text { THRP } \\
(\mathrm{bps})\end{array}$ \\
\hline 50 & 8165 & 5.5 & 3,7 & 32 & 11876 \\
55 & 9124 & 5.75 & 3,7 & 36 & 12694 \\
60 & 9519 & 6 & 3,8 & 38 & 12692 \\
65 & 9942 & 6.25 & 3,8 & 38 & 12726 \\
70 & 10064 & 6.25 & 3,8 & 39 & 12882 \\
75 & 10549 & 6.75 & 3,9 & 40 & 12503 \\
80 & 11865 & 7.75 & 3,9 & 44 & 12248 \\
85 & 13776 & 8.25 & 4 & 51 & 13359 \\
90 & 16140 & 9.75 & 4 & 57 & 13243 \\
95 & 20815 & 11 & 4 & 73 & 15138 \\
100 & 39442 & 18.75 & 4 & 139 & 16829 \\
\hline
\end{tabular}

C.R.: Compression Rate, THRP: Throughput

A.B.T : Average Bytes Transferred

A.T.R : Average Time Required

A.N.U : Average Network Utilization

A.P.S : Average TCP Packets Sent

As can be observed, the less the compression rate, the higher the throughput. This is natural, since the compression takes place real-time and the bandwidth offered is considerably larger than the payload transmitted. Thus, the average network utilization never exceeded the barrier of $4 \%$. Table III on the other hand apposes the results taken from the transmission of 6 different types of medical images, namely Ultrasound, PET, Mammogram, CT, MRI and Retinal. The properties of each image as well as the utilization of the network during their transmission are all cited. For reasons of accuracy and fidelity, each image was transmitted from the client to the server 10 times, adding up to a total of 60 transmitted files.
TABLE III

TRANSMISSION OF MEDICAL IMAGES USING HTTP OVER 3G NETWORKS

\begin{tabular}{ccccccc}
\hline Type & US & PET & MG & CT & MRI & Retinal \\
\hline $\begin{array}{c}\text { DIM. } \\
\text { Size }\end{array}$ & $256 \times 320$ & $384 \times 384$ & $2^{10} \times 2^{10}$ & $2^{9} \times 2^{9}$ & $2^{9} \times 2^{9}$ & $2^{10} \times 2^{10}$ \\
(KB) & 81 & 289 & 423 & 514 & 514 & 1025 \\
$\begin{array}{c}\text { Format } \\
\text { Color } \\
\text { Mode }\end{array}$ & TIFF & DICOM & TIFF & DICOMDICOM & TIFF \\
Bit \\
$\begin{array}{c}\text { Depth } \\
\text { T.T.T. } \\
\text { (sec) }\end{array}$ & 8 & GS & GS & GS & GS & GS \\
$\begin{array}{c}\text { MIN } \\
\text { N.U. }\end{array}$ & $0.24 \%$ & $0.25 \%$ & $0.34 \%$ & $0.33 \%$ & $0.25 \%$ & $0.27 \%$ \\
$\begin{array}{c}\text { MAX. } \\
\text { N.U. }\end{array}$ & $18.55 \%$ & $22.22 \%$ & $22.48 \% 24.95 \%$ & $25.51 \%$ & $26.91 \%$ \\
$\begin{array}{c}\text { THRP } \\
\text { (bps) }\end{array}$ & 36864 & 40127 & 41253 & 40880 & 41281 & 44664 \\
\hline
\end{tabular}

DIM: Dimensions, MG: Mammogram, GS: Grayscale

T.T.T: Total Transfer Time

MIN.N.U: Minimum Network Utilization

MAX.N.U: Maximum Network Utilization

Since the maximum network utilization was $26.91 \%$, the authors deemed it would be purposeful to study the network utilization in terms of the time required for the transmission of the same images and the network throughput using the File Transfer Protocol (FTP). The results are summed up in Table 4 and the difference between the two tables is really intriguing. The authors established an FTP server and uploaded the images using an FTP client. As can be seen in table 4, this process decreased the time required for the transmission of the medical images significantly and increased the network throughput up to 7.5 times as compared with the transmission using the HTTP. Consequently, the transmission of biosignals can take place real time without the need of high bandwidth networks, yet the transmission of medical images requires high bandwidth, while the transmission time fluctuates according to the protocol used for the transmission.

TABLE IV

TRANSMISSION OF MEDICAL IMAGES USING THE FTP

\begin{tabular}{cccccc}
\hline Type & $\begin{array}{c}\text { Size } \\
(\mathrm{KB})\end{array}$ & $\begin{array}{c}\text { T.T.T } \\
\text { 3G } \\
(\mathrm{sec})\end{array}$ & $\begin{array}{c}\text { THRP } \\
\text { 3G } \\
(\mathrm{Kbps})\end{array}$ & $\begin{array}{c}\text { T.T.T } \\
\text { GPRS } \\
(\mathrm{sec})\end{array}$ & $\begin{array}{c}\text { THRP } \\
\text { GPRS } \\
(\mathrm{Kbps})\end{array}$ \\
\hline US & 81 & 2.4 & 270 & 16 & 40.5 \\
PET & 289 & 8.5 & 272 & 59 & 39.2 \\
MG & 423 & 12 & 282 & 83 & 40.8 \\
CT & 514 & 15 & 274.1 & 102 & 40.3 \\
MRI & 514 & 15 & 274.1 & 101 & 40.7 \\
Retinal & 1025 & 29 & 282.7 & 204 & 40.2 \\
\hline
\end{tabular}




\section{DISCUSSION}

The preceding tables summarize the benefits of using $3 \mathrm{G}$ telecommunications technologies in telemedical applications. In this section, along with the further discussion of the results of the measurements, follow different scenarios of the use of the proposed implementation. A number of those scenarios are summarized in Figure 1, which is actually the schematic of the proposed teleconsultation system.

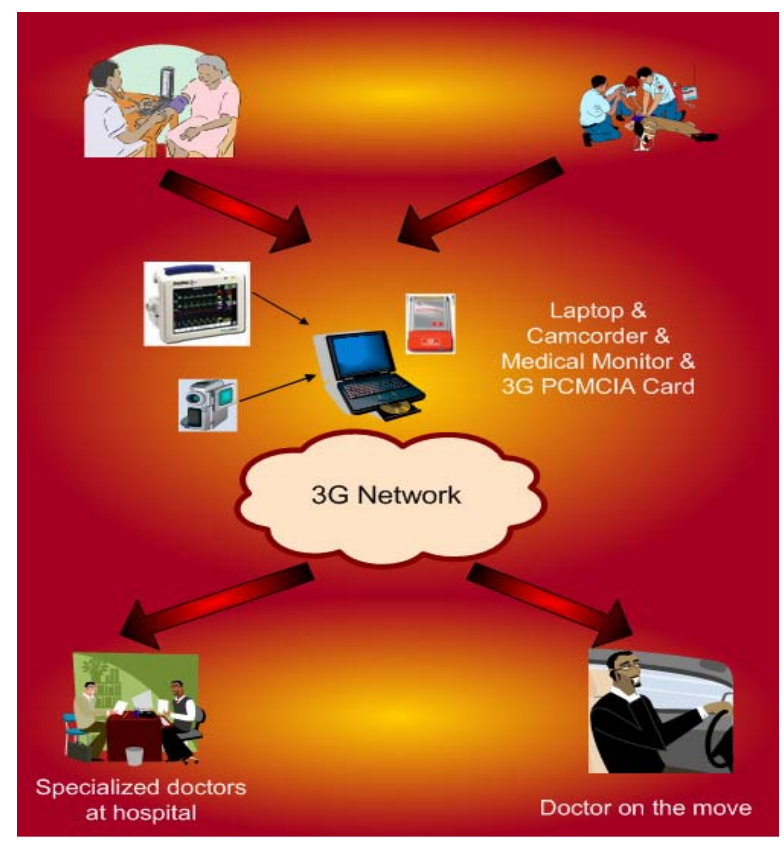

Figure 1: Proposed Teleconsultation System

A quick comparison between the results of the tables point out the significant reduction as regards the time required for the transmission of files from the side of the patient to the side of the expert. The authors were limited to the transmission of biosignals and medical images, yet what is even more intriguing and will constitute the objective of the authors' research is the transmission of bidirectional video. Transmission of medical images is vital since a significant percentage of accidents lead to incorrigible mistakes. For example, in many cases of multi-injured persons, the victims should only be moved by experts and in a specialized manner. The optical perception of the patients from the experts can increase the viability of such accidents. What could be even more helpful is the transmission of real time video. In this case the doctors would have a substantial apprehension of the patient, and the patient would feel more secure just by having a visual contact of the remote doctor.

Figure 1 depicts the transmission of medical data (biosignals, still images and/or video) of the patient either at home or from the accident scene through the $3 \mathrm{G}$ network, to a specialized doctor located either at home (using a fixed line or the $3 \mathrm{G}$ network as well), at the hospital (using the hospital's LAN) or on the move. The capabilities of the proposed infrastructure expand to a wide range of applications of healthcare delivery. Thus, it can be applied to chronic patients, for example diabetic patients, whose glucose levels can be monitored either at home or while on the move and be transmitted to their healthcare providers, as well as benefit the provision of healthcare in rural centers.Young doctors don't want to take full responsibility of the patients. The contact with specialized doctors would significantly uplift these young doctors and reduce patient transportation rates, thus reducing costs.

The tests conducted could have taken place using a $3 \mathrm{G}$ mobile phone with a high quality integrated camera instead of a laptop computer, a web-cam and a PCMCIA card. The use of such a mobile phone would significantly decrease the complexity and the weight of the overall system.

One of the most significant features of the $3 \mathrm{G}$ networks that was stated earlier but was not analyzed thoroughly is the soft hand-over. The transmission of the medical files took place while on the move. The transition from one cell to another was not perceivable by the users, since the laptop equipped with the $3 \mathrm{G}$ PCMCIA card kept contact with more than one base stations simultaneously. Impermanent losses of signals may not be crucial in many real time applications, yet is crucial in cases where healthcare delivery is concerned and human lives are at risk.

\section{ACKNOWLEDGMENT}

The authors of the paper would like to thank Vodafone Greece for their support, both for the hardware and the airtime donated.

\section{REFERENCES}

[1] Congress Office of Technology Assessment, "Bridging Health Care Online, the Role of Information Technologies", OTA-ITC623, Washington D.C., U.S. Government Printing Office, September 1995.

[2] Greek Ministry of Health, National Centre of Emergency Response, Annual Report - 1998, Athens, Aug 1999.

[3] Pavlopoulos S., Kyriakou E., Berler A., Koutsouris D. "Emergency telemedicine applications using mobile and internet communication links - The AMBULANCE Project", Proceedings of EURO-MED NET 98 Conference, Nicosia, Cyprus, pp. 281-282, 1998.

[4] Vern A. Dubendorf, Wireless Data Technologies, John Wiley \& Sons LTD, 2003.

[5] C. Déchaux and R. Scheller. What are GSM and DCS. Electrical Communication, 2nd Quarter 1993.

[6] http://www.gsmworld.com/technology/gprs/intro.shtml

[7] Flavio Muratore, UMTS Mobile Communications for the Future, John Wiley \& Sons LTD, 2001.

[8] G. Mandyam \& J. Lai, Third-generation CDMA Systems for Enhanced Data Services, Academic Press,2002.

[9] www.welchallyn.co.uk/hospital/hospital_catalog.pdf, page 8

[10] Efthyvoulos Kyriacou, Research-Development and Evaluation of an Integrated Telemedicine System for the Remote Monitoring and Diagnosis via Wireless and Wired Communications, PhD Thesis, 2000. 